\title{
Acercamientos a la gestión de la extensión universitaria en Argentina y México
}

Approaches to university extension management in Argentina and Mexico Ramsés Oviedo Pérez ${ }^{1}$

Dalila Emilse Capeletti2

Ezequiel Fabricio Barolin ${ }^{3}$

\section{Resumen}

El presente ensayo reúne dos entrevistas realizadas a los responsables de extensión de dos universidades latinoamericanas. Por un lado, la Universidad Autónoma de Querétaro y por el otro, la Universidad Nacional de Rosario. El objetivo es compartir experiencias y perspectivas, así como el trabajo que se viene desarrollando en dos puntos opuestos de América Latina: México y Argentina respectivamente.

Palabras clave: Extensión, Universidad Autónoma de Querétaro, Universidad Nacional de Rosario

\section{Abstract}

This essay brings together two interviews conducted with those in charge of extension at two Latin American universities. On the one hand, the Universidad Autónoma de Querétaro and on the other, the Universidad Nacional de Rosario. The aim is to share experiences and perspectives, as well as the work that is being carried out in two opposite points of Latin America: Mexico and Argentina respectively.

Keyword: Extensión, Universidad Autónoma de querétaro, Universidad Nacional de Rosario.

\footnotetext{
${ }^{1}$ Licenciado en Filosofía por la Universidad Autónoma de Querétaro (UAQ). Editor de la revista Diseminaciones (UAQ) y Coordinador Académico de Freire, S.C. Correo electrónico: ramsesop@freire.mx

${ }^{2}$ Licenciada en Relaciones Internacionales por la Universidad Nacional de Rosario (UNR). Correo electrónico: dalilacapeletti75@hotmail.com

${ }^{3}$ Licenciado en Relaciones Internacionales por la Universidad Nacional de Rosario (UNR), estudiante avanzado de la Maestría de Estudios Históricos de la Universidad Autónoma de Querétaro (UAQ). Correo electrónico: e_barolin@gmail.com
} 


\section{Introducción}

El presente escrito es fruto del trabajo colectivo que permitió realizar dos entrevistas en tiempos y espacios distintos, para unirlas en un mismo lugar de reflexión. El objetivo es compartir dos experiencias en la gestión de la extensión universitaria en América Latina, que responden a lógicas nacionales diferentes, y de esta forma abrir, a partir de ellas, el debate constructivo y colectivo.

Las entrevistas fueron realizadas a la titular de la Secretaría de Extensión de la Universidad Autónoma de Querétaro (UAQ), México, y su homólogo argentino de la Universidad Nacional de Rosario (UNR). A continuación, transcribimos "libremente" la entrevista realizada a inicios del mes de marzo a la Dra. María Teresa García Besné, de la UAQ. Seguidamente, presentamos las respuestas a similares preguntas hechas al Responsable del Área de Extensión y Territorio de la UNR, Ing. Agr. Santiago Dearma. Finalmente, concluimos con algunos comentarios generales y personales en torno a la temática abordada.

Las preguntas no son exhaustivas y desde la generalidad intentan abordar un amplio abanico de cuestiones como son los actores intervinientes, el financiamiento de las actividades, la comunicación externa, la sistematización y fundamentos teóricos e históricos de las prácticas. La idea de esta propuesta es como lo insinúa el título: un "acercamiento" que nos permita conocer las perspectivas de trabajo en extensión por un lado en México, específicamente en la Universidad Autónoma de Querétaro y por otro en la Argentina, atendiendo al caso particular de la Universidad Nacional de Rosario.

La Universidad Autónoma de Querétaro tiene una larga historia de antecedentes que se remonta al siglo XVI, con la fundación del colegio jesuita de San Ignacio de Loyola y San Francisco Xavier (hasta su expulsión), así como con la creación del Colegio Civil en la segunda mitad del siglo XIX, pero fue hasta 1950 que a iniciativa del Gobernador, Dr. Octavio S. Mondragón, se iniciaron los planes para crear la Universidad de Querétaro, la cual, tras una lucha local movilizada por estudiantes, adquiere su régimen autonómico como tal en febrero de 1959, desde entonces conocida como UAQ.

Actualmente existen 13 facultades, 11 campus distribuidos en varios municipios del Estado, una escuela de bachilleres en la modalidad escolarizada y semi-escolarizada, 24 doctorados, 75 maestrías, 40 especialidades y 81 carreras de licenciatura y 3 técnicas. Según versa en su página web, la Extensión Universitaria tuvo sus inicios en la década de los sesenta con la difusión cultural y artística, pero como veremos, adquirió mayor importancia recientemente. 
En cuanto a la Universidad Nacional de Rosario fue creada en 1968 a través de la Ley 17.987. Su estructura actual es de 12 facultades, 3 institutos de enseñanza media y 1 centro de estudios interdisciplinarios. Cuenta con una superficie edilicia de 68.000 metros cuadrados donde brinda una oferta académica compuesta por 171 carreras de posgrado, 63 títulos de grado, 15 tecnicaturas, 53 títulos intermedios, 26 títulos por articulación con el sistema de educación superior no universitaria y 32 pos-títulos.

Desde el año 2019, el nuevo organigrama de la institución cuenta con 10 áreas de gestión siendo la antigua Secretaría de Extensión el actual Área de Extensión y Territorio.

\section{Entrevista a la Dra. Teresa García Besné}

Es Licenciada en Derecho, pero su pasión por el arte la impulsó a realizar una maestría en Arte (también por la Universidad Autónoma de Querétaro) y posteriormente un Doctorado en Artes Visuales e Intermedia en Valencia. Desde enero de 2019 es titular de la Secretaría de Extensión.

Su actitud es cordial, amena, y una evidente apasionada de su trabajo. Nos recibió en su oficina con la actitud cálida característica de los amigos. Luego de intercambiar opiniones y apreciaciones, tuvimos un diálogo, más o menos parecido al que dejamos por escrito a continuación:

Entrevistador - Vamos a comenzar por lo esencial ¿desde qué año funciona la extensión en la UAQ y cuál es el marco regulatorio legal?

María Teresa García Besné - Históricamente, la extensión inició como una dirección. Como Secretaría de Extensión, habría que revisar bien las fechas, pero si no mal recuerdo funciona como tal desde 1998. O sea, si existía un fuerte interés en que el extensionismo tuviese un papel importante, pero no fue hasta el 98 que se convirtió en secretaria, lo que permitió generar una serie de funciones mucho mayores a las que se pueden generar siendo sólo una dirección.

El extensionismo se encuentra en los estatutos orgánicos, siendo uno de los pilares fundamentales de la labor universitaria, junto con la actividad académica y la investigación. Los tres pilares que sostienen el proyecto universitario.

E.- Y durante el periodo en que la extensión era realizada por la dirección ¿qué actividades de extensión se realizaban?

MTGB -Cuando la extensión se manejaba desde la dirección, todas las actividades de extensión se realizaban de modo intuitivo. No fue hasta la administración de Alfredo Zepeda Garrido, a finales del 2000, cuando esta secretaria recién conformada creó la 
Secretaría de Vinculación que estableció dos líneas muy puntales de prácticas profesionales, y por primera vez se sistematizó el servicio social.

E. - ¿Y por qué le parece tan importante la extensión como pilar de la Universidad? MTGB - Considero que sin el labor del extensionismo, el universitario no tendría una cara al exterior, digamos que es el espíritu de la Universidad. El servicio a la comunidad, al exterior. Lo que da vida al conocimiento profesional.

E.- Recién mencionó al extensionismo como algo diferente a la vinculación social. ¿Podría explicarnos la diferencia?

MTGB - El extensionismo es el deber ser, el espíritu de la labor de universitaria al exterior. La vinculación es una herramienta (desde mi punto de vista), para generar convenios, para generar fondos, para recibir y generar proyectos. El extensionismo es una labor enorme en la Universidad. Un poco en broma, te voy a decir lo que decía el primer director de extensión: Está la academia, la investigación y "todo lo demás”, o sea que todo lo demás era el extensionismo. Y efectivamente, ahí se "metió" en un principio todo lo que no tenía cabida como investigación o docencia. ¿Entonces que entraba aquí? Cultura, servicio social, vinculación con el sector productivo, prácticas profesionales, el deporte, la salud, todo le correspondía a la extensión. Ahora todo ello se encuentra sistematizado y organizado.

E. - La extensión universitaria tiene diferentes aplicaciones en América Latina. ¿Cuál es el papel que desempeña la extensión en México, y específicamente en la UAQ? ¿Cuáles son las prioridades de la secretaria?

MTGB - Hoy por hoy, el extensionismo ha tomado mucho más fuerza y es uno de los trabajos que yo busco fortalecer. Por muchos años, el extensionismo en la universidad se llevó de una manera siendo la principal fortaleza el tema cultural. Estaba soslayado el deporte, la salud y el servicio social (que como conté, hasta hace muy poco se sistematiza). Actualmente la Secretaría tiene 4 direcciones y 4 coordinaciones, lo que la hace más fuerte para llevar su labor de manera concreta y específica. Tenemos una Dirección de Vinculación con el Sector Productivo, ya su nombre dice bastante; la Dirección de Vinculación Social; la Dirección General de Bibliotecas, que es un tema abierto al debate en cuanto a si las bibliotecas deberían estar o no como un servicio de extensionismo; la Dirección de Innovación y Creatividad Cultural, que viene tomando el lugar de lo que fue la Dirección de Difusión Cultural por muchísimos años, pero que hemos decidido modificar tanto en nombre como en actividades.

Como coordinaciones que dependen de estas direcciones están, la Coordinación Editorial, la Coordinación de Librerías Universitarias y la Coordinación de Educación Continua, son tres áreas que saqué de otras dependencias. Por ejemplo, Educación 
Continua dependía de Vinculación Tecnológica, lo que también en términos de nombre, tenía grandes limitantes. La Dirección de Innovación y Creatividad Cultural tomó otra forma que es mucho más inclusiva, es un trabajo que va más allá de difundir cultura, es promover el diálogo y la discusión de temas actuales a través del arte. Toca temas muy importantes que permite el poder trabajar de la mano con vinculación social.

E. - O sea que las prioridades de la Secretaría de Extensión estarían básicamente resumidas en estas cuatro direcciones que también tienen proyectos específicos y metodologías específicas muy concretas.

MTGB - Ejes temáticos diría yo.

E. - ¿Qué son las prácticas profesionales y los servicios sociales?

MTGB. - El servicio social es obligatorio a nivel nacional. Legalmente está establecido para todos los estudiantes de universidades públicas y privadas. ¿Cuál es la diferencia entre servicio social y prácticas profesionales? Básicamente, es que las segundas son necesariamente obligatorias en las áreas de estudios específicas en la que los alumnos se preparan, dirigidas a realizar actividades relacionadas con tu profesión, con lo que estás estudiando; y el servicio social es un servicio dirigido a la comunidad que no necesariamente está vinculado con tu conocimiento profesional. Incluso trabajamos mucho con la metodología aprendizaje-servicio, y pugnamos mucho por la idea de que los chicos del servicio social salgan a trabajar en comunidades. Que hagan un trabajo verdaderamente comunitario y no se quedan en una dependencia gubernamental sacando fotocopias, e insisto, que se encuentre ligado a sus conocimientos profesionales, por ejemplo, puedes ir al campo habiendo estudiado arquitectura, pero allí estás, haciendo zanjas para sembrar.

E. - El servicio social es obligatorio en todas las carreras y en todas las universidades no así las prácticas profesionales que algunas pueden existir o no dependiendo el área de estudio...

MTGB - Exacto. Hay carreras en que las prácticas son obligatorias, quizás el ejemplo más claro es la medicina, no así, por ejemplo, la filosofía. Si no mal recuerdo, la experiencia del servicio social empezó con los estudiantes de agronomía haciendo servicio social para el campo, por ejemplo en Brasil y Argentina, dando a conocer lo que como estudiante de agronomía podían enseñar o compartir y que lo habían aprendido en la universidad. Se trata, en otras palabras, de contribuir a la sociedad.

E. - ¿El servicio social es considerado como actividad de extensión?

MTGB - Efectivamente, son actividades de extensión y son administradas por la Secretaría. El servicio social está regulado por la Dirección de Vinculación Social que 
tiene como función principal su coordinación. Hay una coordinación específica de servicio social, y que regula las prácticas de servicio social, también tenemos un consejo de servicio social coordinado por cada uno de las coordinaciones de las facultades que sesiona una vez al mes. Las prácticas profesionales, por su parte, están a cargo de sector productivo, que también, obviamente depende de la Secretaría.

E. - Pensando en las diferencias entre la universidad pública y la universidad privada ¿Qué destaca en torno a la manera de llevar el extensionismo?

MTGB - Te lo puedo decir de manera muy superficial porque desconozco cómo funcionan las universidades privadas. Pero el compromiso que tiene la universidad pública es mucho mayor que el de una privada. Por ejemplo, mientras que la universidad privada realiza muchos convenios con empresas, nosotros le damos un peso muy importante al trabajo comunitario. De los 18 municipios que tiene el Estado de Querétaro, nosotros tenemos convenio con 13. Existe un programa que se llama verano intensivo, donde un grupo de estudiantes se va por tres semanas a vivir a una comunidad. Nosotros tenemos el compromiso de devolver los que sociedad no da. La diferencia es abismal entre lo privado y lo público, y para nosotros es prioritario.

Para nosotros impera la metodología de aprendizaje-servicio, que se vaya al "campo" a entender el mundo en el que van a desarrollarse o desenvolverse como profesionales después, entender también las necesidades que tenemos como país. El compromiso de devolver a la sociedad lo que nos da.

E. - ¿Con qué actores de la sociedad trabaja?

MTGB - Básicamente trabajamos con empresas, individuos, alumnos, ex alumnos, comunidades. No tenemos la puerta cerrada a nadie, y cualquier ciudadano puede acercarse a trabajar en el servicio social como voluntario o con proyectos propios. Sí tenemos reglas establecidas de trabajo, pero tenemos hasta servicio social voluntario, de personas que hacen literalmente voluntariado, van a trabajar a la sierra o a lugares inhóspitos. Incluso alumnos que a pesar de tener sus créditos completos y sin necesidad alguna de hacerlo porque ya no tiene que cumplir sus horas, participan. Muchos ex alumnos cumplen con proyectos de vinculación social, y también nos generan la posibilidad de hacer proyectos con ellos.

E. - ¿Con qué organismo se vincula principalmente?

MTGB - Dependiendo el área. Vinculación de Sector Productivo con empresa, y el área de Vinculación Social con el área comunitaria. Sin embargo hemos generado, gracias también a que mis directores de áreas son muy jóvenes, promedio 36 años, un proyecto colaborativo, y dado las políticas federales, el nuevo gobierno impulsa las necesidades de trabajar con el sector comunitario. Y nosotros nos vemos envuelto en ello, en parte 
también por las convocatorias federales, tenemos que hacerlo. Pero todas van enfocadas, a lo social, hemos empezado trabajar mucho con Vinculación Sector Productivo, Vinculación Social y Vinculación Cultural en proyectos sociales donde se involucren temas de tecnología ,de medio ambiente, de sustentabilidad, donde nos apoya mucho el ámbito de las ingenierías y las ciencias duras. Y dentro de los sectores que trabajamos de manera muy puntual se encuentra el sector gubernamental, prácticamente tenemos convenio con todos los municipios del Estado. En lo que respecta al sector empresarial, tenemos convenio con la Cámara de Comercio, la CANACO, COPARMEX, que son cámaras que nos permiten llevar proyectos de empresario con el sector del campo y triangular las acciones y actividades para resolver mejor los problemas sociales de nuestro país.

E. - ¿Poseen convenios con otras universidades?

MTGB. - Algunos acuerdos no se concretan y quedan en ideas. La UAQ tiene un peso muy importante en el Estado, tiene una gran relevancia por el nivel académico y por las condiciones de ser la universidad pública más importante. Quizás el ejemplo más claro de trabajo con otras universidades es el tema del río, un trabajo que se hizo de manera transversal y comunitaria. Convenios hay, podría decirse, con todas las universidades pero no todos son aplicados o están funcionando.

E. - Cuando uno piensa en la Feria Internacional del libro de Guadalajara, lo asocia con la Universidad de Guadalajara, cuando uno piensa en el Cervantino, lo asocia con la Universidad de Guanajuato. ¿Hay algunas actividades en la UAQ que tengan esa relevancia?

MTGB. - Hay grandes diferencias, la Universidad de Guadalajara y la Universidad de Guanajuato son universidades del Estado, entonces pueden generar proyectos de gran envergadura en ese sentido. Además, el Cervantino y la Feria son cosas que se salieron de las manos de ambas universidades y lo maneja el Estado. No hay un evento que nos posicione en esa dimensión. Estamos haciendo nuestro esfuerzo por generar el foro de vinculación, que es lo que podemos sacar a la calle de manera general como extensión. Pero tener esos comparativos nos queda grande.

E. - ¿Cuáles son las principales fuentes de financiamiento que existen en la Secretaría de Extensión?

MTGB. - Sin duda los convenios. Desde la administración anterior se generó la sistematización, por decirlo de alguna forma, de los proyectos de vinculación. Por años, la universidad ha tenido un rigor muy importante con los proyectos de investigación pero no lo había en el tema de vinculación. Se generó entonces, una propuesta para sistematizar los proyectos y que se pudieran registrar y se manejaran por un 
reglamento, normas establecidas, etc. A partir de allí se creó el FOVIN, los Fondos de la Inversión para la Vinculación que permite un trabajo de vinculación más controlado y eficiente, podríamos decir que ahora el trabajo se encuentra "normado". Como los profesores registran el proyecto de vinculación en esta Secretaría, nosotros somos capaces de darle un seguimiento particular y saber que están realizando el trabajo y cómo lo están haciendo. Cada dos años re-abrimos la convocatoria.

En principio, se otorgaba una "bolsa" para apoyar por lo menos el inicio de proyecto de investigación (100 mil pesos). La Dirección de Investigación tenía lo propio con una convocatoria de investigación que se llama FOVI. Actualmente se fusionaron las dos en FONDEC, que administra tanto la parte de investigación como la parte de vinculación. Volviendo a la pregunta inicial, los proyectos en su mayoría son con financiamiento externo. Los profesores que hacen vinculación son lo que generan el vínculo con la empresa, con la comunidad, con el gobierno, etc., y traen fondos. Otras fuentes de financiamiento son los apoyos empresariales, otros son los convenios con actores gubernamentales y otros son los propios fondos que genera la universidad.

E.- En términos administrativos, ¿hay algún órgano de control que le dé seguimiento al uso de estos recursos?

MTGB.- Sí, Contraloría. Aquí todo el dinero que ingresa en la universidad va a cuentas de la Secretaría de Finanzas. Cada proyecto que se registra tiene que estar aprobado por el Comité de Vinculación para que nosotros podamos generar la solicitud de la apertura, si no, no se apertura la cuenta.

E.- ¿O sea que si ustedes no la aprueban, el proyecto no se inicia?

MTGB.- Nosotros tenemos un comité interno que es la CREP, conformado por los directores de vinculación e innovación y la secretaria de Extensión. Ese comité revisa que los documentos se encuentren completos para su registro. El siguiente paso es el Comité de Vinculación, que está conformado por los coordinadores de vinculación de cada facultad. Es ese comité el que aprueba o niega el registro del proyecto en cuestión. De ser aprobado, aquí se genera la solicitud a la Secretaría de Finanzas, para que le dé apertura a la cuenta, aperturada la cuenta, los fondos entran a esta secretaría con el nombre del proyecto y nosotros vamos generando los recursos que la investigación necesita.

E.- ¿Existe algún modelo teórico explícito en el funcionamiento de la Secretaría?

MTGB.- La actividad que realiza la Secretaría es tan grande que cada una de las direcciones tienen modelos muy específicos. Yo te diría que el modelo que sigue la Dirección de Vinculación Social es el de "Aprendizaje-Servicio". Por ejemplo, el sistema particular que estamos utilizando en la Dirección de Vinculación con Sector 
Productivo es el de "Formación Dual", que es un modelo alemán que estamos intentando "tropicalizarlo" para las necesidades del país, en el sentido de que los estudiantes trabajen en empresas privadas (en palabras sencillas). O sea, entiendan el mundo real en una organización empresarial. Hemos comenzado con dos empresas y en el área de ciencias duras, pero estamos intentado hacerla extensiva a campos de las ciencias sociales.

Lo que te comentaba de la Dirección de Investigación e Innovación Cultural que modificó el nombre de Dirección de Difusión Cultural, está relacionado a un cambio de la visión en la forma de trabajar también. No nos estamos limitando a difundir cultura o a formar públicos, sino a generar proyectos críticos que, a través del arte, den respuesta a las problemáticas sociales del país. En breve, por ejemplo, vamos a tener la visita de Ophelia Pastrana que va a realizar una charla-debate en el contexto de los feminismos en México. Pero no sólo ello, constantemente se están realizando iniciativas semejantes que interactúen con las problemáticas de la sociedad que habitamos. Por ejemplo, también te puedo mencionar que tuvimos un encuentro bastante importante de ciencia y tecnología, otros sobre cultura de paz, que más servicios (que también lo hacemos), han sido laboratorios de experimentación.

E.- Hace ratito mencionaba que sí ha habido un proceso de sistematización con la creación de reglamentos y normas, ¿pero hay un trabajo de análisis e investigación sobre las prácticas de extensión en la localidad y en los campi?

MTGB.- El registro de proyectos nos está permitiendo sistematizar el trabajo de vinculación y conocer lo que se está realizando. La realidad es que los profesores hacían vinculación y esta secretaría ni siquiera se daba del todo enterada. Ahora, tenemos un comité conformado por Secretaría Académica, la Secretaría de Extensión y la Dirección de Investigación y Posgrado justamente para conocer y tratar sobre los temas que implican a las tres áreas. La realidad es que el extensionismo ha cobrado mucho más relevancia en los últimos diez años, anteriormente teníamos muy sueltas las cuerdas de todo, aunque ahora tampoco las tenemos tan atadas. Conocer permite mayor control, más eficiencia y también nuevas ideas. Bajo esta concepción de "conocer" es que estamos digitalizar nuestros sistemas de trabajo como Secretaría.

E.- ¿El repositorio digital de la UAQ tiene alguna relación con la extensión?. Le comento por la experiencia argentina: hay una ley, la 26899 de Creación de Repositorios Digitales Institucionales de Acceso Abierto que salió hace unos años atrás en donde todas las universidades tiene que tener su repositorio digital y la producción de la universidad se socializa gratuitamente en estos repositorios, por ejemplo, las tesis. 
MTGB.- No, mira, aquí obviamente sí tenemos el repositorio de tesis. Bellas Artes en algún momento hicieron un repositorio de temas de cultura o de artistas.

E.- De hecho hicieron su propia editorial...

MTGB.- Exacto. Pero institucionalmente no tenemos eso, lo cual me parece muy interesante y me parece que se podría considerar a futuro.

E.- Sería algo muy positivo. Una pregunta que me surge es ¿cómo distinguen un proyecto de vinculación y otro de investigación?

MTGB.- Es una cuestión que dejamos bajo criterio del profesor. El maestro dice: "esto es vinculación y lo voy a registrar a la Secretaría de Extensión”, o "esto es investigación y lo registro en la Dirección de Investigación”. Si bien son cuestiones que pueden solaparse o confundirse, la importante es que el proyecto pueda realizarse.

E.- En cuanto a lo que es la comunicación de la Secretaría de Extensión explícitamente, ¿cómo lo hacen, a través de redes sociales? ¿Cuáles? ¿Cómo se manejan administrativamente en ese sentido?

MTGB.- Es una cuestión bastante importante para mí. Yo provengo de humanidades y mi área específica es el arte, entonces obviamente todo lo visual para mí es "un tema”. Lo primero que hice al ocupar mi cargo, fue promover una imagen consensuada de la Secretaria con sus variantes para cada dirección. También se promocionó un video institucional para dar a conocer los proyectos y actividades de la Secretaria. Cada área sube su propia información tanto a las redes de la secretaría, que son todas: Instagram, Facebook, etc., como a la página, que está lo más actualizada que puede estar una página en la universidad. Existe una persona encargada solamente del trabajo de redes, así como de tener la página lo más ordenada posible. Todo lo que hacemos lo socializamos y lo enviamos a las redes de la universidad (que prácticamente es la que más impacto tiene a nivel estatal), y a prensa de la universidad.

E.- Sobre el asunto del espacio, ¿cómo es la relación con los diversos campi de la UAQ? ¿Cómo ha sido el crecimiento, por ejemplo, del Museo XIMHAI y el Museo de la Estudiantina? De alguna manera pareciera ser que Extensión está también apropiándose y creando más espacios.

MTGB.- Las relaciones con los campi afortunadamente es muy positiva, especialmente con algunas áreas en concreto, como Vinculación Social y Dirección General de Bibliotecas, Con Vinculación Sector Productivo hay campus en los que podemos trabajar de manera mucho más puntual. En mi caso particular, he tratado de hacerlo de manera mucho más directa con el campus Amazcala de Ciencias Naturales: el tema de los quesos y la producción del rancho en general. Pero se trata de procesos que se van profundizando y consolidando en el tiempo. 
Artes y Oficios de Educación Continua también ha tenido un trabajo digno de ser destacado. Yerett Oliveri, mi coordinadora del área merece todo el reconocimiento porque ha catapultado de una manera increíble ese tema. Nosotros tomamos la Escuela de Artes y Oficios con unos seis talleres, actualmente llevamos más de veinte y se ofrecen cada semestre, reproduciéndoles en diferentes campus. Hemos estado trabajando en Concá, en Jalpan, en Cadereyta, en San Juan del Río, estamos intentando lo mismo en Tequisquiapan, en la Casa de Independencia. Este último caso, era un lugar que tomamos vacío con la idea de que los grupos universitarios pudieran practicar y ensayar, pero ahora se ha convertido en una especie de casa de cultura, aunque no es nuestra intención.

El cuanto al Museo XIMHAI, que todavía es muy pequeñito, lo estamos tratando de impulsar debido a que no tenemos un museo de ciencia en Querétaro. No es un museo de ciencias como yo quisiera que lo fuese, el lugar es muy pequeño y obviamente carece de muchisimo. Sin embargo lo abrimos para impulsarlo, para crecer, para encariñarnos y poder empezar a andar el proyecto y permitir así, el apoyo externo. Lo abrimos con poco dinero, pero con mucho entusiasmo.

Respecto del Museo de la Estudiantina, tiene la intención de cubrir a todo tipo de público. De conformarse como un espacio más hacia la sociedad, para que conozca la tradición universitaria. No obstante, la idea de abrir estos espacios no significa que hayamos cerrado la puerta para propuestas contemporáneas. Bajo esa idea, adquirimos un piano, como Secretaría de Extensión y estamos dando conciertos con los pianistas más reconocidos del INBA cada mes. Entonces estamos tratando de cubrir todos los frentes en la medida de nuestras posibles condiciones.

E.- ¿La UAQ participa en redes nacionales e internacionales de extensión para llevar a cabo sus actividades?

MTGB.- Sí, claro, una de las más importantes es la ANUIES. Aun que actualmente cada vez es más largo el plazo entre una sesión y otra es la red más importante de universidades. Además, ANUIES no solo tiene la red de extensionismo sino que también tiene la de servicio social y la de innovación. De manera aleatoria, participamos con algunas redes internacionales.

E.- ¿Piensa que la UAQ podría vincularse en algún momento con otras redes internacionales?

MTGB.- Sí. Cuando nos hemos relacionado ha sido para proyectos específicos, o sea no hay una continuidad como la que existe en la ANUIES en la que si formas parte, tienes sesiones regulares y acuerdos. Si hay una red internacional con estas características estaríamos encantados de participar de manera regular. En la mayoría de redes en la 
que hemos entrado ha sido por sector productivo o vinculación social y con alguna propuesta específica, pero se han tratado de temas intermitentes.

E.- Para finalizar: a lo largo de todos los años que lleva en la UAQ, ¿cómo percibe esa historia del extensionismo en la UAQ?

MTGB.- Debo reconocer que en el área de extensión me involucré de una manera más puntual hace pocos años. Yo he estado siempre en el área de cultura, y en esa área no necesariamente todos los casos pertenecen a extensión. Mi cercanía con el extensionismo se profundizó a partir de la Dirección de Difusión Cultural y ahora al frente de la Secretaría. Sin embargo, las transformaciones en el extensionismo se dieron gracias al arduo de mis antecesores, con intención de reafirmar el papel del extensionismo. Debo destacar que fue en la administración anterior a cargo de la maestra Magali Aguilar donde se sentaron las bases, y con Gilberto Herrera como rector, donde se consolidaron, para un extensionismo más estructurado. Sólo una buena organización puede producir resultados eficientes.

\section{Entrevista a Ing. Agr. Santiago Dearma}

Es Ingeniero Agrónomo, no alcanza los treinta años y desde comienzos del 2018 se desempeña como Responsable del Área de Extensión y Territorio de la Universidad Nacional de Rosario.

Debido a las medidas sanitarias de aislamiento social obligatorio la entrevista se realizó por correo electrónico. Quizá por ello carece de los detalles propios de un encuentro no virtual como la posibilidad de la repregunta, pero lo cierto es que tiene absoluta precisión, una enorme generosidad y también, para quienes formamos parte de esta Universidad y en particular de este equipo de trabajo, una gran carga emotiva por la convicción presente en cada una de sus palabras.

Entrevistadora - Para comenzar, ¿desde qué año funciona la extensión universitaria en la Universidad Nacional de Rosario (UNR)? ¿Existe un marco legal que la encuadre y la diferencie de la vinculación social? ¿Cuál? ¿Qué perspectiva tiene de la extensión universitaria?

Santiago Dearma - Si bien no ha sido ajena a cambios y discusiones en torno al sentido que esta tiene, la extensión universitaria ha estado presente desde la creación de la Universidad Nacional de Rosario en 1968. Hay que tener presente que su creación fue llevada adelante durante el gobierno de Onganía, lo cual supuso una tensión entre la tradición reformista que expresaba la Universidad Nacional del Litoral -UNL- (que fue la estructura sobre la que se constituyó la UNR) y el gobierno de facto. Por otra parte, en esa etapa inicial lo que existía era un Departamento de Extensión Universitaria, que incluso funcionó en el período democrático que va del 73 al 76. Pero 
es recién a partir del proceso de normalización universitaria que se inicia en el 84 que se crea la Secretaría de Extensión Universitaria, con un Secretario designado por el Rector. La primera autoridad de la flamante Secretaría fue el Dr. Ovide Menin, quien en algunas partes de su libro Pedagogía y Universidad recupera experiencias de trabajo en el marco de la Secretaría.

En relación a la cuestión prescriptiva, tanto en los estatutos anteriores a la normalización (cuya base normativa responden a la tradición reformista de la UNL) como los nuevos marcos legales, contemplan a la extensión universitaria como uno de los pilares del sistema universitario. De todos modos, aún en el Estatuto vigente, la cuestión de la extensión se plantea de un modo demasiado genérico y en tal sentido no se advierte una importante discontinuidad en torno a la idea de vinculación (tal vez en el marco de época en que se discutió nuestro Estatuto aún no había una separación en las prácticas y representaciones de quienes desarrollaban la extensión). De todos modos, como sabemos quiénes gestionamos y hacemos extensión, existe un importante hiato entre la dimensión prescriptiva que se expresa en los marcos normativos, y la esfera descriptiva de las prácticas concretas. En efecto, hasta hace menos de un año extensión y vinculación se expresaban en diferentes secretarías. Si bien puede haber un debate donde podamos identificar las perspectivas dialógicas con la idea de extensión y los modelos transferencistas o difusionistas con la noción de vinculación tecnológica, la política del actual Rector se orienta hacia una fuerte integración de las áreas y campos de actuación universitaria. Nosotros desde la gestión concebimos a la extensión como diálogo de saberes. Es decir, nos distanciamos de perspectivas que consideran a la Universidad como la usina exclusiva de un saber que se transfiere a periferias de no saber. Por el contrario, creemos que la diversidad sociocultural supone diversidad epistemológica, esto es, reconocer otros saberes que pueden ser significativos para dar respuestas a las problemáticas que atraviesan las sociedades complejas, y por ello es importante dialogar con ellos, siendo la Universidad por su naturaleza, el ámbito que debe alojar dicha pluralidad.

E.- ¿Qué entiende por extensión universitaria y por qué cree que es tan importante? SD.- La extensión universitaria además de ser una función sustantiva de nuestras Universidades Públicas Nacionales es además, a mi modo de ver, una idea de militancia en el marco de las mismas. Partiendo de esta base, hoy consideramos a la extensión como un proceso educativo transformador de las prácticas de formación de nuestros estudiantes, proceso además que vincula una idea de Universidad ligada estrechamente a los sectores populares y que trabaja y piensa sus avances basándose en las problemáticas de la sociedad que la contiene. 
Considero que la misma es tan importante debido a que otorga la posibilidad de formar individuos críticos y contextualizados con una realidad que, como siempre digo, es la que muchas veces se encuentra de la ventana del aula para afuera.

E.- La extensión universitaria tiene diferentes aplicaciones a lo largo de todo Latinoamérica, ¿̨cuál es el papel que desempeña la extensión en la UNR? Actualmente, ¿cuáles son las prioridades de la Secretaría?

SD.- Hoy día, y gracias a una apuesta de gestión del actual rector de la Universidad, la extensión cumple un rol fundamental en nuestra Universidad, ya sea en el pensar de la formación de nuestros estudiantes como en la manera a través de la cual nos vinculamos con organizaciones de la sociedad civil.

Las prioridades hoy están fijadas en el repensar la práctica de formación estudiantil, tratando de incorporar aún más la formación en contexto de los y las estudiantes y en la profundización de nuestras prácticas de extensión junto a muchas organizaciones de base territorial.

E.- ¿Cuáles son los principales ejes temáticos en los que trabaja la Secretaría de Extensión? (Derechos humanos, medio ambiente, salud comunitaria, etc.) ¿El arte y las humanidades tienen vinculación con el modelo de extensión que promueve la UNR? SD.- Claramente al hacer un análisis de los ejes en torno a los cuales giran las propuestas de extensión en nuestra Universidad existe una multiplicidad de propuestas que trabajan ligadas a diferentes temáticas. Hoy el género, los derechos humanos, el medio ambiente son temáticas claves a la hora de pensar la extensión y la Universidad consideradas transversales a nuestras prácticas. Hoy no vemos la posibilidad de pensar una práctica de extensión que no contemple dichas temáticas, por ende diría que son la matriz de pensamiento que hoy rige nuestra forma no sólo de ver a la extensión sino a la misma Universidad. A ellos se suman temáticas diversas como ser la salud comunitaria, el acompañamiento a espacios de la economía social y popular, etc.

El arte así como las humanidades son claves a la hora de pensar la extensión crítica, como herramientas de transformación social. Hoy quienes pensamos la extensión desde un punto de vista crítico creemos en estas herramientas como el motor de muchas de nuestras prácticas de contención y acompañamiento de personas en situación de vulnerabilidad. Lo vemos en las experiencias que hoy se llevan adelante tanto con niños, niñas, jóvenes como con personas privadas de su libertad. Entendemos el arte como una forma más de interacción e integración comunitaria.

E.- ¿Qué son las prácticas sociales obligatorias? ¿La UNR tiene pensado incluir este tipo de prácticas en su gestión? ¿Cómo? ¿Cuándo? 
SD.- La propuesta de prácticas sociales educativas (PSE) implica pensar en experiencias curriculares desarrolladas en territorio, en donde las y los estudiantes universitarios puedan experimentar instancias de trabajo y aprendizaje en interacción con múltiples actores. Se trata de propiciar instancias de aprendizaje situado, en contexto, producto de la acción sobre él. El/la estudiante aprende participando en prácticas con la comunidad, en contextos vinculados con aspectos de su futura práctica profesional. Esto supone el desarrollo de competencias concebidas como saberes en acción, como praxis que articula conocimiento y práctica. Adaptando el concepto de competencias y ajustándolo al contexto de las prácticas sociocomunitarias decimos que en estas situaciones los estudiantes construyen capacidades complejas y potenciales para actuar en contextos comunitarios reales, integrando y usando conocimientos y procedimientos de las disciplinas y actitudes o valores solidarios, de manera estratégica y con conciencia ética y social.

Estamos actualmente trabajando en el armado de una propuesta con el objetivo de incorporarlas en nuestra Universidad. Ni bien retomemos la actividad vamos a presentar una propuesta para que pueda ser analizada y trabajada por el conjunto de nuestra comunidad. Cabe destacar que en el armado de la propuesta consideramos fundamental el aporte que podamos generar a través de la participación y aportes de todos los miembros de las diferentes unidades académicas y la participación de todos los claustros de la Universidad.

E.- ¿Considera que existen diferencias entre la Universidad Pública y Privada al respecto de la extensión universitaria? ¿Cómo visualiza la relación de la UNR con la sociedad?

SD.- Hemos observado a lo largo del tiempo que existe una concepción de la extensión en muchas Universidades muy ligada a la responsabilidad social la cual difiere de la concepción de extensión que hemos definido como matriz a la hora de pensar la extensión en nuestra Universidad que es la extensión crítica. Esta última se basa principalmente en reconocer la existencia de una diversidad de saberes y la bidireccionalidad de las prácticas de extensión. Cuestiones que desde los ámbitos privados son muchas veces difíciles de pensar, ya que muchas veces desde estos espacios se concibe a la extensión como un proceso de transferencia, de saber, de tecnología, de ideas. Estos procesos no dejan lugar a la interpelación por parte de otro sino que lo considera como mero receptor de un mensaje.

E.- ¿Cómo se organiza la Secretaría? ¿Cómo se conforma el equipo de trabajo?

SD.- Desde hace unos meses, tras la asunción del actual rector, el Lic. Franco Bartolacci, el organigrama de la Universidad quedó delimitado en Áreas de Gestión. Extensión y 
Territorio es una de ellas. Dentro de nuestra área hemos creado 5 direcciones, las cuales representan no sólo una manera de organizarnos, sino que además son el reflejo de lo que hoy consideramos como fundamental a la hora de pensar la función de la extensión en nuestra Universidad.

Las 5 direcciones son: Dirección de desarrollo territorial, Dirección de desarrollo social, cultural y productivo, Dirección de vinculación institucional, Dirección de convocatorias y Dirección de formación y capacitación.

El grupo de trabajo es un grupo muy heterogéneo. Por las trayectorias de cada una y cada uno de los que conformamos hoy el equipo del Área de Extensión y Territorio, por las disciplinas profesionales, por nuestros recorridos particulares en el plano de la gestión pública; y por múltiples cuestiones más que hacen hoy de esa heterogeneidad, a mi modo de ver, la principal fortaleza de este equipo.

E.- ¿Quiénes son los participantes de las propuestas de extensión universitaria? (Estudiantes, docentes, graduados, administrativos, miembros de organizaciones o instituciones) ¿Cómo se promueve su participación?

SD.- Hoy los participantes son en su mayoría estudiantes, muchas y muchos promotores de grandes ideas que son llevadas adelante en el marco de proyectos y programas de extensión en nuestra Universidad. Es de destacar la participación de miembros de organizaciones sociales e instituciones también en las propuestas. Creo que tanto la participación estudiantil como de miembros de movimientos y organizaciones sociales, son hoy de las mayores riquezas al momento de evaluar las propuestas que año a año se presentan. Docentes, no docentes y graduados también tienen participación en ellas. Cabe mencionar que hoy día las propuestas son en su totalidad dirigidas por equipos docentes que a su vez realizan el acompañamiento durante la duración de las propuestas.

Desde hace ya varios años, la manera de promover la participación de los miembros de nuestra comunidad en procesos de extensión, vienen siendo las convocatorias que anualmente se inscriben en el marco de nuestro trabajo de gestión. Convocatorias que han ido variando con el correr de los años. Actualmente nos encontramos en un proceso de revisión de las mismas con el objetivo de imprimirles muchos de los objetivos que nos hemos planteado como ejes de nuestra gestión para los próximos cuatro años: tales ejes se basan principalmente en la posibilidad de curricularización de nuestras prácticas extensionistas y la sintonía de estas prácticas con las problemáticas territoriales actuales. 
E.- ¿Con qué actores externos a la Universidad se vincula principalmente? (Organizaciones sociales, organismos del estado, empresas, etc.) ¿Qué tipos de intervenciones se llevan a cabo con ellos?

SD.- Al hacer un repaso de las organizaciones con las cuales hoy existe una vinculación muy cercana de la mayoría de las propuestas se identifica una cada vez más marcada participación de organizaciones de base territorial, ya sean estas clubes barriales, bibliotecas populares, merenderos, organizaciones sociales, etc. Actualmente hemos encontrado una gran sintonía de trabajo con varias dependencias del estado, mayormente aquellas que tienen una fuerte impronta de territorio, como ser centros de salud, centros de convivencia barrial y distritos; y también con muchas instituciones escolares, tanto de nivel inicial, primarias y secundarias.

Muchas de las intervenciones tienen que ver con el acompañamiento de diferentes procesos de integración con niños, niñas y jóvenes en situaciones de gran vulnerabilidad que de no ser por la contención que hoy encuentran en estas organizaciones de base territorial, muchos y muchas de ellas quedarían excluidos y excluidas de cualquier círculo social de contención.

E.- ¿Cuáles son las fuentes de financiamiento para las actividades de extensión? ¿Qué requisitos existen para acceder a ese financiamiento? ¿Existen órganos de control para el seguimiento y evaluación de las actividades de cada gestión universitaria?

SD.- Hoy una gran cantidad de actividades de extensión son financiadas a través de estas convocatorias que comentaba anteriormente, con presupuesto propio de nuestra Universidad. También hay que mencionar el enorme esfuerzo que hacen día a día quienes participan de estas actividades para funcionar muchas veces bajo la modalidad de autogestión, encontrando en el equipo del área acompañamientos desde otros puntos de vista.

Los requisitos principales es que las prácticas se encuentren en el marco de alguna de las Facultades o Escuelas de nuestra Universidad, que la dirección la pueda llevar a cabo un docente rentado, que se garantice la participación de estudiantes en el marco de las prácticas y la existencia de una contraparte, es decir, de alguna organización de la sociedad civil.

La evaluación de las propuestas tiene una fase presencial previa al otorgamiento del subsidio, instancia en la cual tiene la posibilidad de participar la totalidad del equipo para defender la propuesta frente a un jurado integrado por referentes de la extensión de nuestra Universidad, de Argentina y Latinoamérica. Una vez concluida cada una de las prácticas también hoy se exige la presentación de informes finales para evaluar la relación de las propuestas con lo aprobado en su momento. Además de esto existe 
también un programa de acompañamiento a esas prácticas en cuestiones logísticas y de formación principalmente.

E.- ¿Cuál es la forma de trabajo? ¿Se basan en algún modelo teórico-metodológico explícito? ¿Cuál?

SD.- La forma de trabajo se basa principalmente en fomentar la participación activa de todos los miembros del equipo no sólo en la puesta en marcha sino en la toma de las definiciones en el marco de nuestro trabajo cotidiano. La extensión crítica es el paradigma dentro de la extensión que hoy nos identifica grupalmente y como Universidad, ello nos propone pensar a diario nuestros abordajes comunitarios y sobre todo las formas a través de la cual nos vinculamos.

E.- ¿Realizan algún proceso de sistematización sobre las experiencias universitarias? ¿Tienen trabajo de análisis e investigación sobre las prácticas realizadas en el territorio local y regional?

SD.- La sistematización de experiencias es una práctica que hemos incorporado en nuestra cotidianeidad gracias al gran aporte de referentes como Humberto Tommasino y Oscar Jara y demás compañeros y compañeras que acompañan a nuestro equipo desde ya hace varios años y que sin dudas nos han nutrido de prácticas como estas y muchas más. Una gran falencia que muchas veces identificamos en quienes formamos parte de gestiones tan dinámicas como las universitarias es la imposibilidad de darnos muchas veces el tiempo suficiente para parar la pelota y pensar en el recorrido de nuestras prácticas.

Sin embargo, hace un tiempo hemos presentado lo que para nosotros significa un orgullo como equipo, la sistematización de experiencias de extensión en la Universidad Nacional de Rosario de los últimos 6 años. La misma se materializó en una recopilación de experiencias de gestión en coordinación del ex Secretario de Extensión Universitaria de la U.N.R., el Ing. Juan Manuel Medina y el ex ProRector de Extensión de la Universidad de la República (Uruguay), Humberto Tommasino con participación de todos los miembros de la entonces Secretaría de Extensión Universitaria. Este material pone en valor un gran trabajo que se identifica con una forma de pensar la extensión en nuestra Universidad y rescata todo lo construido junto a muchos y muchas que pensamos a la UNR trabajando junto a los sectores populares de nuestra sociedad.

E.- ¿Cómo es la relación con las unidades académicas de las UNR?

SD.- En nuestra Universidad existe desde hace ya varios años lo que denominamos el Consejo Asesor de Extensión, el cual está formado por los representantes de las áreas de extensión de las 12 Facultades y las 3 Escuelas Medias de nuestra Universidad. Ésta 
es considerada por nosotros una gran herramienta a la hora de pensar la democratización de las políticas de gestión de la extensión y la construcción colectiva de muchas propuestas que nos han llevado hoy a tener una gran sintonía con lo desarrollado en cada una de las Facultades y Escuelas. Dicho esto creo que existe una muy buena relación entre quienes trabajamos en extensión en nuestra Universidad.

E.- ¿Cómo manejan la comunicación hacia afuera de la Universidad? ¿Qué relevancia tienen las redes sociales digitales y medios impresos para la extensión universitaria? SD.- Hoy contamos con un gran equipo de comunicación dentro del área que trabaja codo a codo con la gestión central de la comunicación de la Universidad. Rescatamos en cada una de nuestras prácticas el valor de la comunicación de lo que hacemos con el principal objetivo de contagiar a que cada vez seamos más los que pensemos una Universidad vinculada directamente a las problemáticas sociales actuales.

Lo que se juega en torno a la comunicación de la extensión o en la extensión, es la capacidad para generar y propiciar espacios que habiliten instancias de reflexión de la palabra y del conocimiento y la acción, que redunden en aprendizajes colectivos a la par que logren desarrollar interés por la participación del sistema universitario en estos contextos.

Las redes sociales digitales son hoy nuestra principal herramienta de comunicación ya que nos permiten por sobre todo sostener el vínculo cotidiano con quienes trabajamos. Sin embargo, como comentábamos anteriormente, no dejamos de darle valor a los medios impresos los cuales nos permiten difundir más allá del tiempo experiencias y recorridos.

E.- ¿Cómo piensa que podría vincularse la extensión universitaria con otras universidades? ¿La UNR participa en redes nacionales e internacionales de extensión? ¿Cuáles? ¿Cómo?

SD.- Actualmente gracias a las herramientas de comunicación existe una vinculación casi cotidiana con áreas de extensión de Universidades de nuestro país y de toda Latinoamérica. Además de ello formamos parte de la Red Nacional de Extensión Universitaria (REXUNI), de la comisión de extensión de la Asociación de Universidades del Grupo Montevideo (AUGM) y de otras redes como ser la Unión Latinoamericana de Extensión Universitaria (ULEU). En todas tenemos una participación activa a través de nuestra representación institucional pero por sobre todo a través de la participación en actividades, congresos y encuentros que se realizan en el marco de las mismas.

E.- Ya para finalizar, a lo largo de todos los años que lleva en la UNR, ¿cómo percibe la historia de la extensión universitaria en Rosario? 
SD.- A mi modo de ver, es de destacar el gran trabajo que se viene llevando adelante en materia de extensión universitaria en nuestra Universidad desde hace ya varios años, trabajo que es fruto del esfuerzo de muchos compañeros y compañeras que día a día le ponen el cuerpo a lo que nos proponemos; y por una firme decisión política de nuestros rectores de apostar a una universidad ligada directamente a los sectores populares de nuestra sociedad. Ha habido un marcado avance en la manera de pensar la extensión de nuestra Universidad, ello debido a definiciones de gestión y por sobre todas las cosas conceptuales que nos posicionan desde un lugar de la interpelación diaria del quehacer de nuestras prácticas.

Hoy seguimos escribiendo la historia de la extensión en la UNR, rescatando y valorizando el recorrido que nos trajo hasta acá. No hay un solo día en que no pensemos en lo mucho que aún nos queda por construir como universitarios miembros de una sociedad que espera estemos siempre a la altura de las circunstancias, una sociedad que nos demanda emparentar a nuestra Universidad cada vez más con un contexto que nos reclama, una sociedad a la cual sabemos muy bien no podemos defraudarle.

\section{A modo de cierre}

Son numerosas las reflexiones que se disparan a partir de las entrevistas realizadas, aristas de futuros debates y cuestionamientos al interior de las diversas prácticas de extensión. La primera de ella refiere al papel que ha adquirido la extensión a partir de las últimas décadas en las diferentes universidades. Si bien, la misma existe jurídicamente enmarcada desde hace tiempo, ambas universidades han dado un impulso a su práctica en los últimos años, sosteniendo la importancia de la sociedad en el vínculo bidireccional. En el caso de la $\mathrm{UAQ}$, se destaca la idea de "devolver a la sociedad" algo que se recibe de ella y conocer las necesidades que existen en el medio. Precisamente, se trata de una universidad de provincia empero resulta representativo su trabajo de vinculación-educación-investigación con distintos sectores sociales. Por ejemplo, con el paso de los años ha ido encontrando un arraigo con comunidades de la Sierra Gorda Queretana, sosteniendo abiertamente proyectos que, bajo la figura de brigadas, voluntariados o intervenciones psicosociales, atienden las problemáticas de esas poblaciones. A partir de ahí la UAQ ha redimensionado las bases epistémicas de su modelo de extensionismo. En el caso de la UNR lo que se sostiene es la idea de producir un conocimiento diferente al que se otorga tradicionalmente, es decir, un conocimiento dialéctico, resultado del saber dado y recibido en las prácticas de extensión (premisa que Paulo Freire ya planteó y aquí se hace manifiesta). No obstante, mientras que en 
México las Prácticas Profesionales y el Servicio Social son obligatorios (según establecen sus leyes en la materia) y forman parte de la extensión universitaria, en Argentina no es parte de la misma. En cuanto al Servicio Social, como trabajo "voluntario" paradójicamente de carácter obligatorio en el caso mexicano, no existe en Argentina como tal y menos como parte curricular. El sistema de extensión es estrictamente de carácter voluntario, siendo libre su participación. Analizar el nivel de participación es una cuestión pendiente máxime si se toman en cuenta los rasgos de sujeto político en jóvenes universitarios y las nuevas formas de ciudadanización en entornos postmodernos.

En lo que respecta a la financiación, hay que reconocer un gran trabajo de articulación con organismos privados y gubernamentales por parte de la Universidad Autónoma de Querétaro, respondiendo a la dinámica de su funcionamiento. Sus finanzas dependen de los subsidios estatales y federales, de los ingresos de las licenciaturas, maestrías, doctorados, diplomados y cursos, de los acuerdos vigentes y proyectos que establece con entidades privadas. En el caso de Argentina, la Universidad obtiene sus recursos principalmente del gobierno nacional, por lo cual sus actividades se ven condicionadas por esta partida. Queda pendiente analizar cómo ampliar este margen de maniobra por parte de las Universidades argentinas, y rescatar, quizás, las medidas que se implementan en México. $Y$ es que el recorte al sector educativo mexicano en los últimos presupuestos de egresos de la federación (PEF) ha obligado a optimizar el uso y aprovechamiento de recursos. El ideal no sería comparar las medidas de sobrevivencia económicas, ya de por sí difíciles en la conjunción de agentes decisores, sino cómo los sistemas universitarios se reorganizan para mantener en pie la mayoría de sus proyectos prioritarios de vinculación social.

Otro de los ejes a analizar está relacionado con el proceso de sistematización de las prácticas extensionistas. Sistematizar no sólo es clasificar, catalogar, ordenar datos e informaciones. Como afirma Oscar Jara en su libro La sistematización de experiencias: práctica y teoría para otros mundos posibles, sistematizar es una actividad reflexiva que todos hacemos al recuperar organizadamente la práctica con el objetivo de interpretar críticamente las experiencias vividas para extraer sus aprendizajes y compartirlos. En este sentido, creemos que es muy interesante el trabajo de sistematización crítica que llevó adelante la Universidad Nacional de Rosario en conjunto con el ex ProRector de la Universidad de la República (Uruguay), el cual podría ser replicado en México. Es probable que existan universidades mexicanas que realicen periódicamente procesos de sistematización pero, en cualquier caso, vemos que es importante convertir a este proceso en una regla de dinamismo y no en excepción. 
En cuanto a las actividades de extensión, son diversas y variadas en ambas casas de estudios. Lo destacable es la interacción con la sociedad que supera el mero aspecto cultural, antaño asociado a la extensión y hoy ampliamente considerado sólo uno de sus elementos. Aspecto cultura que demuestra en ambos casos, un compromiso social, un "arte militante" (o comprometido) que va más allá de la observación pasiva y de la formación de públicos y cumple una función de denuncia, de movilización, de involucramiento colectivo. A su vez, ambas universidades se apoyan en las TICs con el objetivo de comunicar su trabajo, comunicar que no es sólo difundir sino fundamentalmente entrar en diálogo con la sociedad a partir de estas nuevas herramientas electrónicas.

Finalmente, cabe destacar la interacción entre los diversos campi de la UAQ y las unidades académicas en la UNR, puesto que es fundamental el diálogo y la participación colectiva a la hora de construir consensos en torno a las modalidades de trabajo en extensión. En este sentido, el grado de institucionalización alcanzado por la universidad argentina a partir de la creación del Consejo Asesor que representa a los secretarios y secretarias de todas las facultades y escuelas medias que la componen, es un interesante desafío para las otras casas de estudio. Se considera por ello que es un desafío la activación de un marco de participación colaborativa, en donde lo que se adquiere y reproduce es un tipo de racionalidad crítica e intercultural.

Con tales menciones, estamos solamente "punteando" algunos elementos que están presentes en ambas entrevistas para abrir el debate a futuros acercamientos y reflexiones conjuntas. ¿Es factible una práctica similar al Servicio Social en Argentina? ¿Qué implicancias tienen el factor económico en las cuestiones de extensión? ¿Qué connotaciones negativas y positivas se desarrollan alrededor de la obligatoriedad de las prácticas? ¿Cómo puede hablarse de una universidad con protagonismo transformador si la base de sus recursos públicos es frágil y constantemente conflictiva por el "estira y afloja” con el Estado? ¿Qué papel juegan en las redes de cooperación universitaria las actividades de extensión? ¿Cuáles son las nuevas prácticas de extensión que están emergiendo en el contexto de la pandemia global por el coronavirus? Pensar la gestión de la extensión no es sólo planificar y ejecutar una serie de actividades repetibles sino que requiere de la apuesta cotidiana de los gestores al diálogo y consenso colectivo por el tipo de universidad que queremos alcanzar. Por eso creemos lo que señala Pablo González Casanova en su libro La universidad necesaria del siglo XXI: vincular el conocimiento técnico y científico con el histórico-político y con las armas epistemológicas y teóricas, morales y críticas de una educación humanista, y de una democracia incluyente es el único proyecto que puede asegurar la viabilidad del 
proyecto universitario. El momento histórico en el que vivimos amerita repensar las condiciones de posibilidad de dicho proyecto. Pero, por supuesto, hay un modelo de universidad presente en las políticas de extensión que se llevan adelante en cada una de las instituciones y está claro en ambos casos que la sociedad es parte constitutiva de la formación profesional. 